\section{Handbook of Cyanobacterial} Monitoring and Cyanotoxin Analysis

\section{J. Meriluoto, L. Spoof, G.A. Codd (eds.), 2017 John Wiley \& Sons Ltd., West Sussex, UK \\ ISBN: 978-1-119-06868-6 \\ Pages: 576; $€ 168,00$}

Cyanobacteria are impacting widely on the daily activities of the general public. They are increasingly recognized as agents having possible adverse effects on water resources, on health of human and/or animals and therefore should be monitored and managed. To fill existing gaps this valuable handbook which is edited by three highly respected experts in the areas microbiology, algae biochemistry, metabolomics, and environmental science provide reviews, practical methods and standard operating procedures.

Scientists from both university and industry contributed a series of chapters summarizing and introducing the reader to Cyanobacteria (Section I and II), reviewing sampling, monitoring including risk management (Section III), and toxins plus other bioactive compounds in Cyanobacteria (Section IV). These sections are followed by a comprehensive survey of state-of-the-art analytical methods to characterize different metabolites in Cyanobacteria (Section V) and methodological considerations (Section VI). Section VII gives several standard operating procedures around various aspects of Cyanobacteria analysis.

The style of this book is not only informative, but also a joy to read. It is well produced and illustrations are given in a very adequate way including a bibliography following each chapter. Therefore, the book should be part of any library of Cyanobacteria microbiologist and cyanotoxin chemist. Furthermore, the book provides very interesting material to be purchased by those with a much broader interest in algae chemistry and ecology.

Stefan Martens

Food Quality and Nutrition Department, Research and Innovation Centre Fondazione Edmund Mach, San Michele all'Adige, Italy 